\title{
The Frequency of Anomalously Red Galaxies in SDSS Clusters
}

\author{
O. Shearman ${ }^{1,2,5}$ and K. A. Pimbblet ${ }^{1,2,3,4}$ \\ ${ }^{1}$ School of Physics, Monash University, Clayton, VIC 3800, Australia \\ ${ }^{2}$ Monash Centre for Astrophysics (MoCA), Monash University, Clayton, Victoria 3800, Australia \\ ${ }^{3}$ Department of Physics, University of Oxford, Denys Wilkinson Building, Keble Road, Oxford OX1 3RH, U.K. \\ ${ }^{4}$ Department of Physics and Mathematics, University of Hull, Cottingham Road, Kingston-upon-Hull, HU6 7RX, U.K. \\ ${ }^{5}$ Email: odshe1@student.monash.edu
}

(Received October 10, 2013; ACCEPTEd August 30, 2014)

\begin{abstract}
We present a systematic photometric search for spectroscopically confirmed anomalously red galaxies members of 748 low redshift clusters between $0.03<z<0.17$ from the SDSS-C4 cluster catalogue (Miller et al. 2005). For each cluster we spectroscopically determine cluster membership, construct a colour-magnitude diagram and fit the red sequence using a robust bi-weight fit. We define an "anomalously red galaxy" as having a $(g-r)$ colour of greater than $3 \sigma$ redward of the fitted cluster colour-magnitude relation. We find that of 7485 galaxies at $r \leq 17.77$ in $(g-r), 7$ galaxies are anomalously red -0.0935 per cent of all galaxies in our sample. We show that two of the red outliers are caused by red contamination from nearby sources and are therefore not intrinsically anomalous red. However, 5 have no underlying cause to be so red and we speculate that they may have a high internal dust content. These intrinsically red galaxies are rare - comprising no more than 0.0668 per cent of all cluster galaxies. Most are morphologically early type galaxies, with a few probable late type galaxies that are viewed edge-on and one low surface brightness late type. One of our anomalously red galaxies appears to be a dust-shrouded starburst and we speculate that this may be a unique galaxy amoungst this galaxy set.
\end{abstract}

Keywords: galaxies: clusters: general, galaxies: elliptical and lenticular, $\mathrm{cD}$, galaxies: photometry, galaxies: stellar content, Hertzsprung-Russell and C-M diagrams

\section{INTRODUCTION}

The Colour-Magnitude Relation (CMR; Visvanathan \& Sandage 1977; Bower, Lucey, \& Ellis 1992; also known as the red sequence) describes the tight (with a scatter typically less than $0.1 \mathrm{mag}$ at optical wavelengths) and Universal correlation between (mainly) early-type cluster galaxies on the colour-magnitude plane. The CMR has been found to be primarily caused by a metallicity effect (Kodama \& Arimoto 1997), although this was later refined by Terlevich et al. (1999) who modelled the CMR as a metallicity caused relation with fluctuations caused through age variations (see also Worthey 1994; Kodama, Bower, \& Bell 1999).

Many recent studies have revealed that the CMR is not a homogenous population. Although the brighter galaxies might be mostly morphologically elliptical or S0 (lenticular), there exist a significant population of comparatively red late-type galaxies within the CMR (e.g. Masters et al. 2010a; Wolf et al. 2009; Lee, Bell, \& Somerville 2008; Goto et al. 2003; Poggianti et al. 1999; see also Cortese \& Hughes 2009; Mahajan \& Raychaudhury 2009; Masters et al. 2010b; Deng, Chen, \& Jiang 2011; Crossett et al. 2013; Ko et al. 2013). It may be the case that optical colours are insufficient to differentiate between truly quenched systems and starforming systems - especially at the upper end of the cluster galaxy mass function (Cortese 2012). Indeed, it seems the case that quenching (environmental or mass driven) must take place prior to morphological transformation to permit the existence of such a red spiral population.

Yet the origin of cluster CMRs is further complicated by the existence of very - suggestively anomalously - red galaxies. By this, we mean galaxies whose colour and colour uncertainty place them significantly above (i.e. redward of) the established CMR by $>3 \sigma$. Our own past work, shows the existence of such anomalously red galaxies (which we herein abbreviate to ARGs). For example, Pimbblet et al. (2002) show a very red galaxy in the Abell 22 colour-magnitude plane but do not pass a comment on its presence. More recently, Jensen \& Pimbblet (2012) described a small set of ARGs in a study of 45 intermediate X-ray luminosity galaxy clusters taken from the Sloan Digital Sky Survey (SDSS; Abazajian et al. 2009). Many of the ARGs were found to be due to saturation effects from nearby objects (stars and other galaxies), whilst the majority of the remainder were 
suggested to have dust-affected colours. However, Jensen \& Pimbblet (2012) found evidence for a handful of truly intrinsically red galaxies that are uncontaminated by nearby sources that lay significantly above the CMR.

In this work, we set out to create the first ever systematic search for ARGs in galaxy clusters from SDSS. We hypothesise that these types of galaxy are intrinsically rare and we therefore seek to address the questions of exactly how rare they are, and what precisely causes their red colours (how often is it due to nearby objects and how frequent are they simply intrinsically red - perhaps due to dust reddening?). We aim to address the question of whether these galaxies are truly quenched (so called red-and-dead), or whether they could be dust-enshrouded starbursts through use of SDSS spectroscopy. In Section 2, we present our dataset of 748 SDSS clusters and observational parameters that we work with to perform this analysis. In Section 3, we analyse our clusters by fitting their CMRs and searching for any ARGs contained within. Section 4 presents our results of this search and we discuss the cause of the redness in these galaxies.

\section{DATA}

For our study, we make use of the Sloan Digital Sky Survey (SDSS) Data Release 7 (DR7; Abazajian et al. 2009) as our basis spectroscopic and photometric galaxy catalogue. The power of SDSS is contained in its wide areal coverage: a key factor that is needed to turn up multiple examples of the very rare class of galaxy that we aim to find.

Additionally, we use the $\mathrm{C} 4$ cluster catalogue of Miller et al. (2005) to identify SDSS clusters within DR7. Although other choices of cluster catalogue are possible for this study, the $\mathrm{C} 4$ catalogue is both up-to-date and highly complete for all classes and types of clusters of galaxies at low redshifts which we contend is more than sufficient for our present investigation.

From the cluster catalogue, we extract cluster galaxies from the SDSS database by taking all galaxies within $\pm 3 \sigma$ of each mean cluster recession velocity given by Miller et al. (2005) and a radial limit of $3 h^{-1} \mathrm{Mpc}$. Although somewhat a crude approach in comparison to caustic methods (cf. Alpaslan et al. 2012 and references therein), this extraction process will return members that are unlikely to be infalling galaxies (cf. Figure 5 of Pimbblet et al. 2006 who show that this extraction is highly similar to a caustic approach using a mass model). Many of our clusters have small numbers of members. It is not 100 per cent possible to rule out the likelihood that some of the members of groups with low multiplicities have incorrectly included field galaxies due to a poor computation of the velocity dispersion. We do, however, note that Robotham et al. (2011) demonstrated a good correspondence of low multiplicity groups with mock datasets in their friends-of-friends group catalogue for the GAMA survey. Therefore we are confident that with our higher multiplicity, our rate of interlopers will be smaller.
For each galaxy, we obtain petrosian magnitudes (from which we derive our colours), positions, redshifts and all pertinent associated uncertainties. The filters utilised are the $\mathrm{g}$ and $\mathrm{r}$ band, later using the $\mathrm{g} \& \mathrm{i}$ band to make a more definitive evaluation of the galaxies redness; the use of the petrosian magnitudes giving a robust measure of the total magnitude of each galaxy. Finally, we note that the limiting magnitude of spectroscopy in SDSS of $r=17.77$ corresponds to $M^{\star}+1$ for our typical cluster at $z=0.08$. Our range of cluster redshifts means that this limiting magnitude will vary strongly over our full redshift range $(0.03<z<0.17)$. We return to this point in Section 4.

\section{ANALYSIS}

For each cluster we construct a colour-magnitude diagram for the spectroscopically confirmed members. To fit the red sequence of each cluster, we use the robust bi-weight fit method presented by Beers, Flynn, \& Gebhardt (1990; see also Pimbblet et al. 2002) and we refer the reader to those works for full details of that method. In brief, it fits the data to find the intercept of the red sequence, $c_{b i}$, the biweight fit estimator, such that

$$
c_{b i}=M+\frac{\sum_{u_{i}}\left(x_{i}-M\right)\left(1-u_{i}^{2}\right)^{2}}{\sum_{u_{i}}\left(1-u_{i}^{2}\right)^{2}}
$$

where $\mathrm{M}$ is the sample median and $u_{i}$ is:

$$
u_{i}=\frac{u_{i}-M}{c(M A D)}
$$

and $\mathrm{c}$ is the tuning constant (Beers et al. 1990) which we set to $\mathrm{c}=6.0$ following Beers et al. (1990) to give $c_{b i}$ a high efficiency. The Median Absolute Deviation (MAD) is simply

$$
M A D=\text { median }\left(\left|x_{i}-M\right|\right) .
$$

Once fitted, we systematically searched each galaxy cluster for ARGs by finding galaxies whose photometric uncertainty placed them $>3 \sigma$ above the fitted red sequence for their given magnitude. An illustrative example of this process is shown in Figure 1 where we depict the colour-magnitude plane of Abell 1809 and have fitted the red sequence to recover a single ARG at $r=17.401$

\section{RESULTS AND DISCUSSION}

Out of 7845 galaxies that are bona fide members of 748 clusters brighter than $r=17.77$, we find 7 fit our criteria of being anomalously red; some 0.0935 per cent of the total cluster population. Figure 2 displays the images and spectra of these ARG from our investigation, along with plots showing the CMR for each cluster.

Out of our sample of seven, two have close companions that affect their photometry, as confirmed by inspection of the pixels contained in the segmentation maps of these images. These are caused by bright nearby stars and neighbouring foreground or background galaxies (Figure 2). There exists 


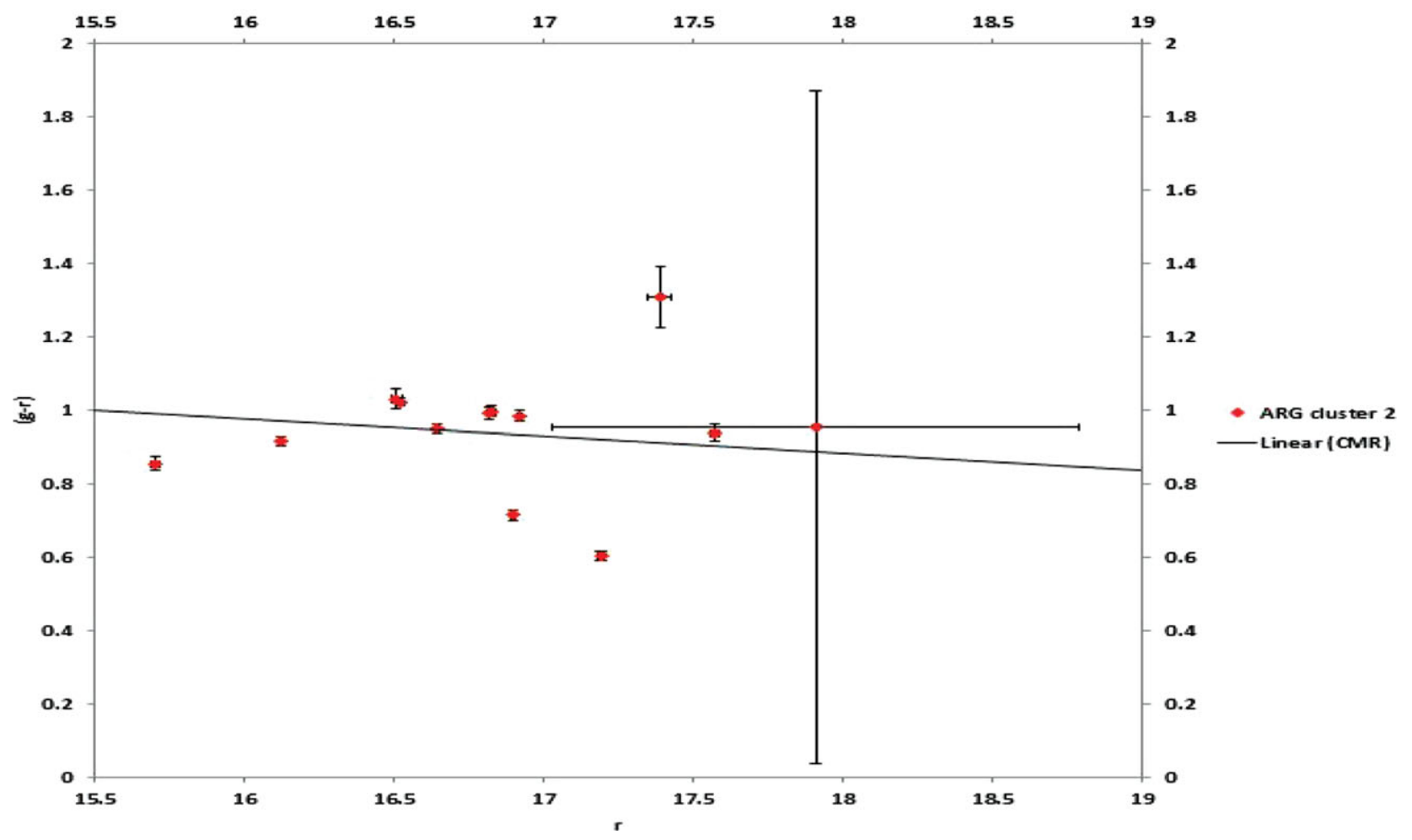

Figure 1. Example colour-magnitude plane (corresponding to ARG 1 in our sample) of one of our galaxy clusters (Abell 1809; SDSS-C4 1001) which depicts the fitted red sequence and an ARG that is $3.92 \sigma$ above the fit, this includes the uncertainty on the measured colour.

the possibility that any of these ARG could be field galaxies that are red due to their dusty nature, though this is unlikely as; the work done in Ruel et al. (2013) shows that when investigating small numbers of member galaxies unbiased velocity dispersions can be obtained, however increased systematic error is also observed. The use of the petrosian model magnitudes from SDSS throughout this work enables overlay image maps to be interrogated for flux contamination. These overlap maps reveal that both ARGs 1 \& 3 suffer from contamination and therefore cannot be considered as truly anomalous in their colours compared to the red sequence. ARG 7 is a borderline contamination case, but the size of the SDSS spectral aperture does not appear to be affected by the close neighbours of this particular galaxy. However, most of our sample appears to be genuinely red. We comment on these cases below.

The first of these is ARG 2 which is a very red, morphologically elliptical galaxy. It has a typical spectrum with no emission and strong 4000 Angstrom break (ARG 6 is a further example of this). The spectrum does not reveal anything particularly strange about ARG 2 or 6 though. It may be the case that they simply have a high dust content. To rule out that this is caused by nearby sources (explicitly, the Milky Way), we used dust maps from Schlegel et al. (1998) to confirm that the Galactic extinction does not vary on the local scale of the ARG or its parent cluster. Hence we conclude that any dust present will likely be an intrinsic property of the ARG itself.
To determine if the red colours are consistent with the spectra, we construct a representative sample of 'normal' CMR galaxies from the clusters we have used in the parent sample. We select all galaxies within $0.1(g-r)$ of the fitted CMR with $16.7<r<17.4$ - i.e. covering the full range of ARG magnitudes - to do this. Both the star formation rate and the specific star formation rate of the comparison sample and these ARGs are statistically indistinguishable.

ARG 4 presents arguably the most interesting ARG case: a potential dusty star-forming galaxy, as suggested by the $\mathrm{H} \alpha$ line both in the spectra and the derived equivalent widths from Brinchmann et al. (2004). The SFR and SSFR for ARG4 are significantly higher than for the control sample, supporting the notion that this is indeed a star-forming galaxy shrouded by dust.

ARG 5 is somewhat enigmatic. It has a very strong Magnesium absorption line which could be due to sky subtraction during processing (note the sky noise spike). However, the derived SFR and SSFR for this galaxy are also significantly higher than for the control sample, suggesting that it may be in the same dust-enshrouded class of ARG4.

ARG7 has a SFR that is significantly higher than the control sample as well, but its SSFR is fairly typical. It may be the case that there is some contamination from pixels from the blue source nearby to this object, but in this case, ARG7 will require followup observations to fully determine the cause of its intrinsically high (apparent) redness. 


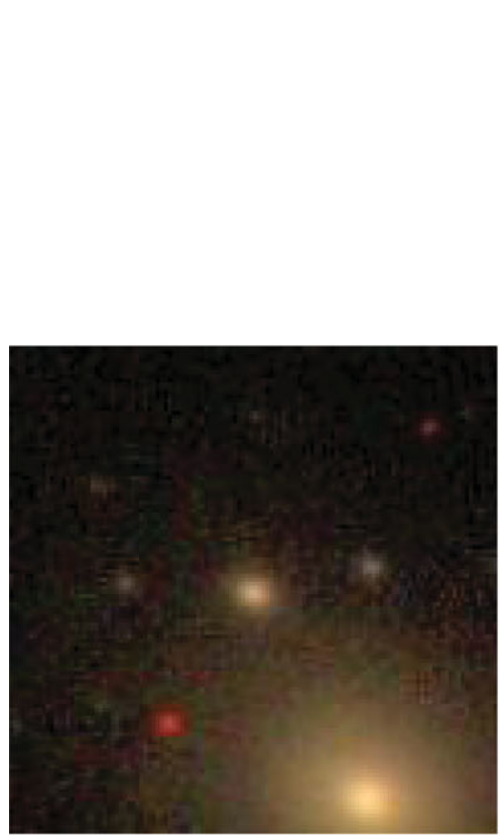

$\mathrm{RA}=208.27980, \mathrm{DEC}=5.15538, \mathrm{MJD}=52375, \mathrm{Plate}=855$, Fiber $=507$
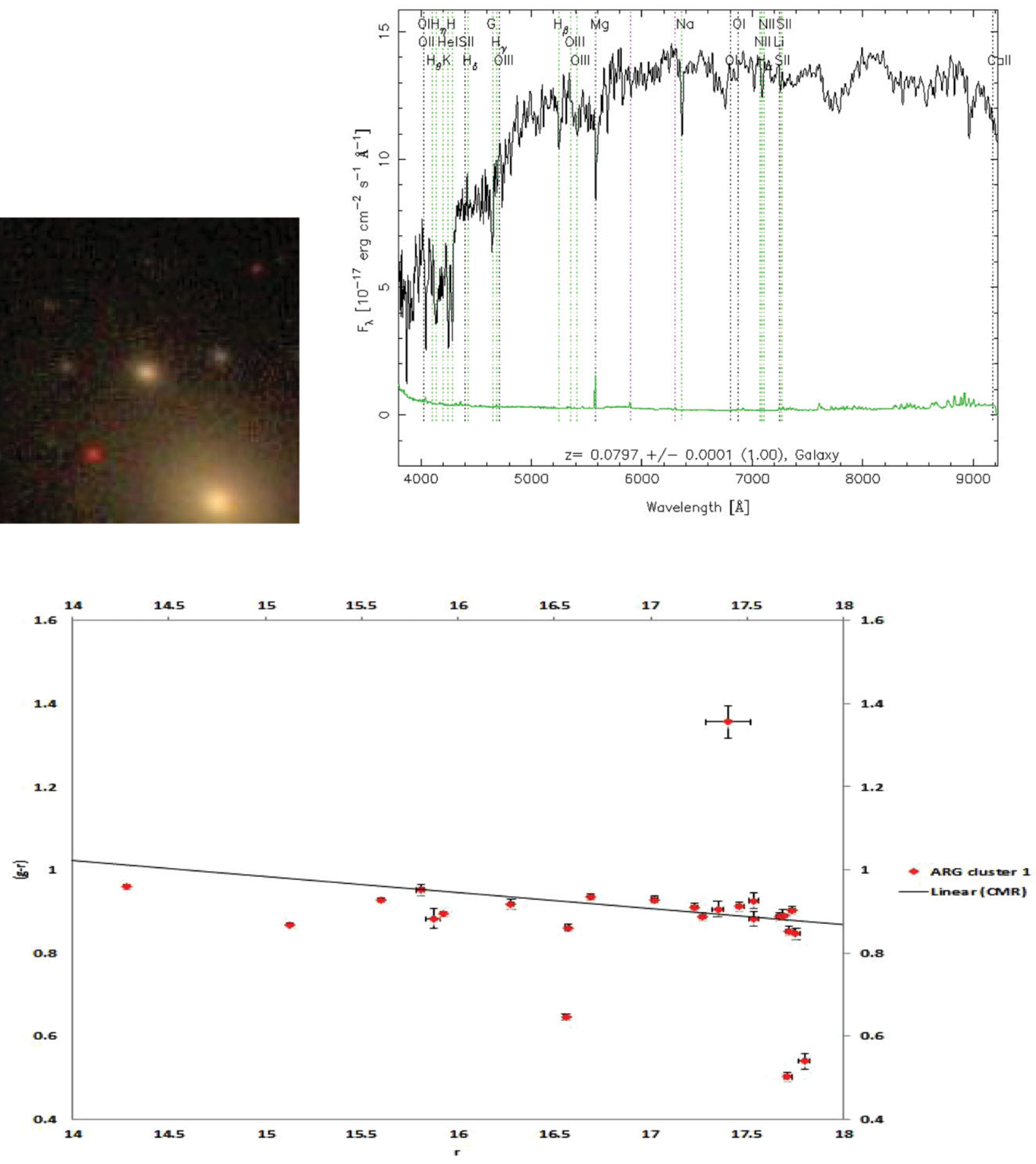

ARG 1

Figure 2. Images and spectra of all ARG found. Each image is 120 by 120 pixels sourced from the SDSS database and each spectra displays the common absorption lines together with the RA, Dec, and SDSS observation tags along the top. Also displayed is the galaxies in each cluster plotted with their corresponding CMR, wavelengths are observed frame. Data on these galaxies is summarised in Table 1.

Finally, we noted above that there was a significant range of $M^{\star}$ values across that range of our cluster redshifts. If we were to apply a common absolute magnitude cut off to our sample of $r=-21.06$ which corresponds to the nominal
100 per cent redshift completeness of SDSS at $r=17.77$ and $z=0.14$ (the redshift of our highest ARG), then we are left with only ARG 4 . This corresponds to 0.0004 per cent of all galaxies brighter than this absolute limit. Changing this to 
$R A=118.65919, D E C=39.17741, M J D=52201$, Plate $=544$, Fiber $=350$

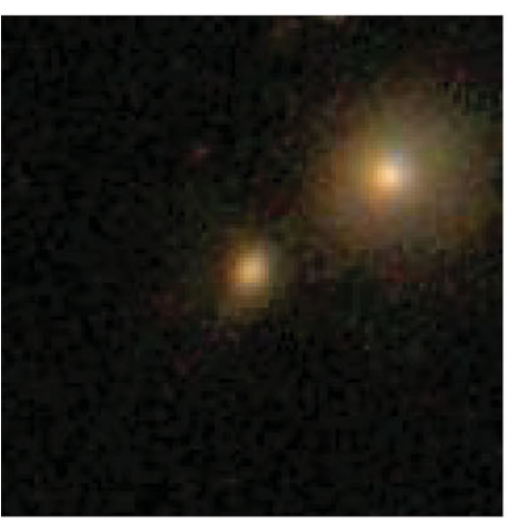

image 2

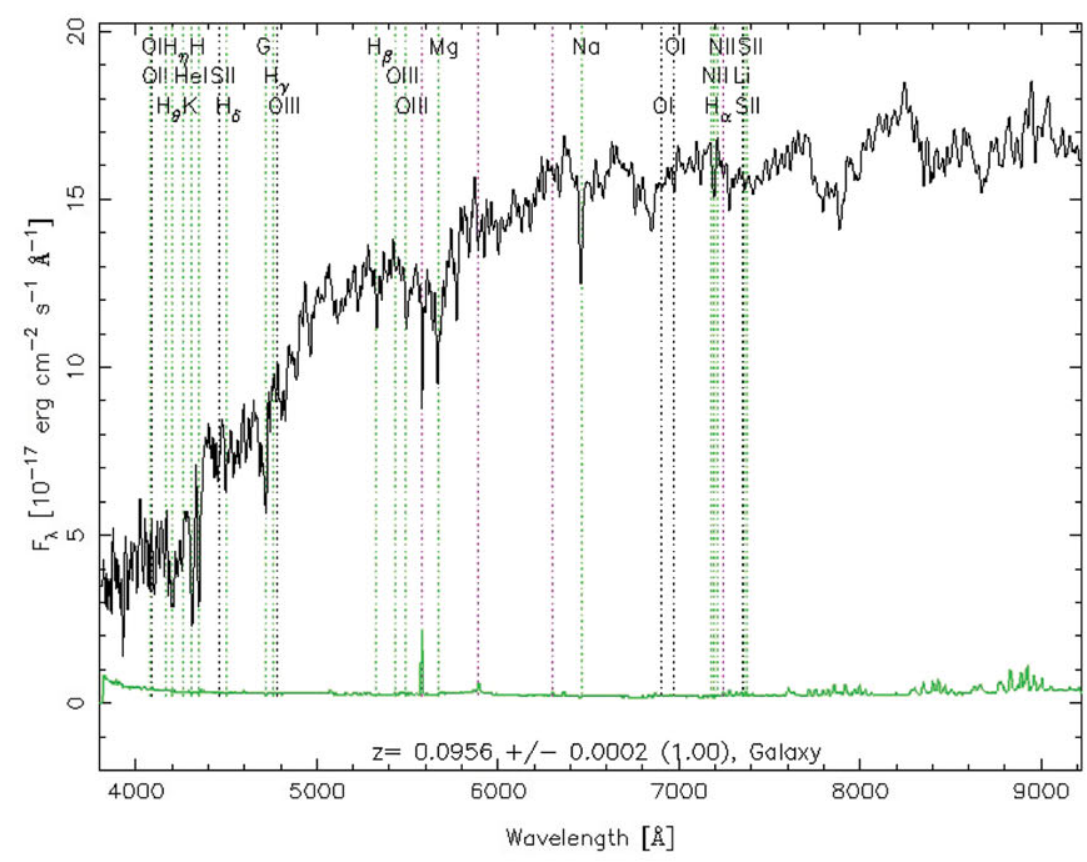

spectrum 2

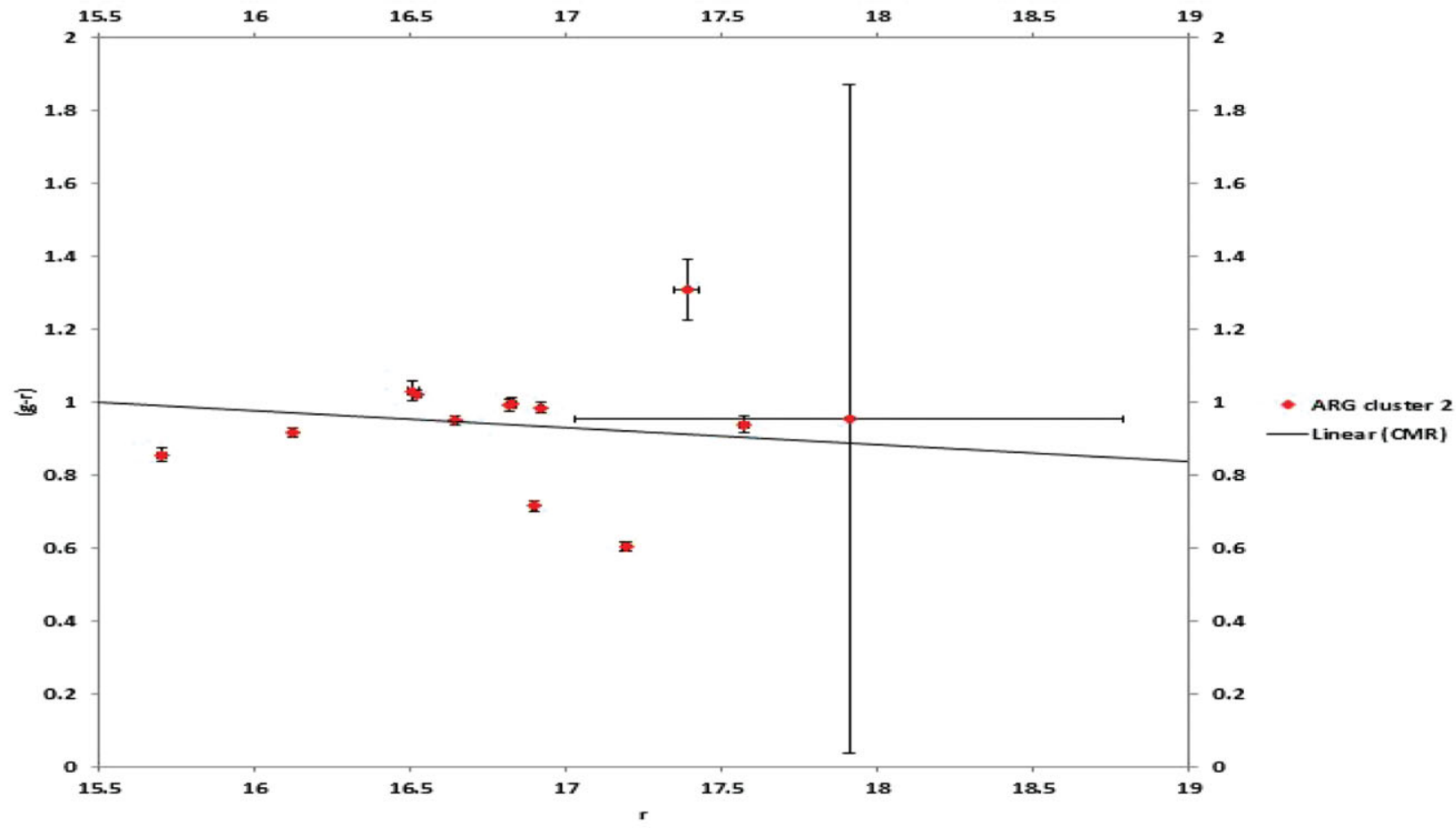

ARG 2

Figure 2. (continued). 
$R A=18.81845, D E C=0.18873, M J D=51789$, Plate $=398$, Fiber $=428$

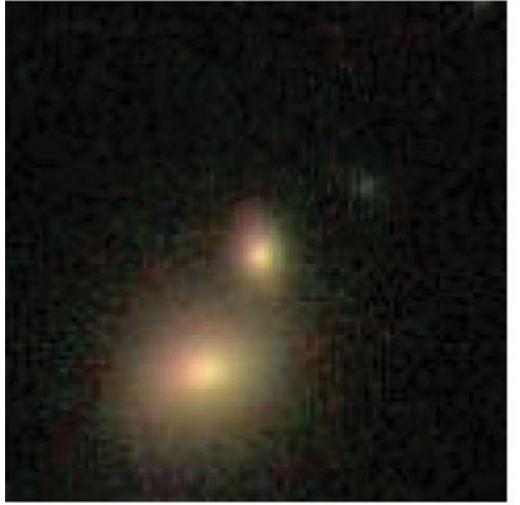

image 3

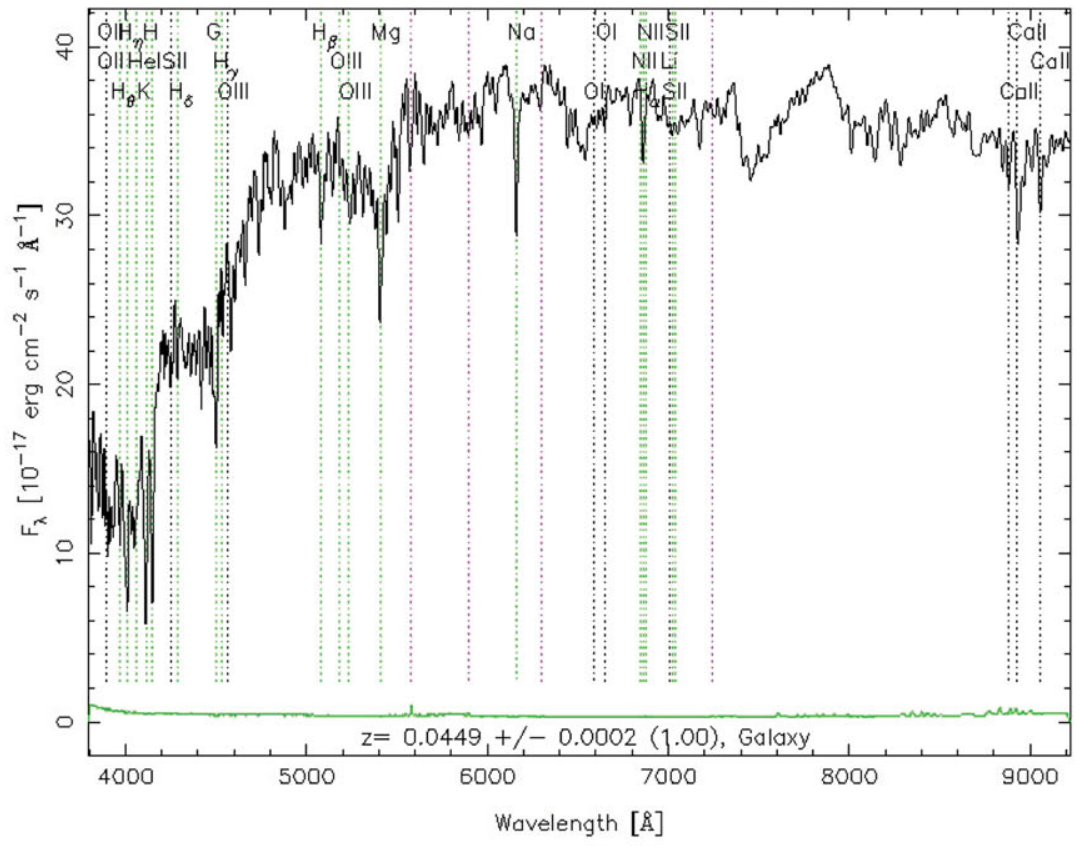

spectrum 3

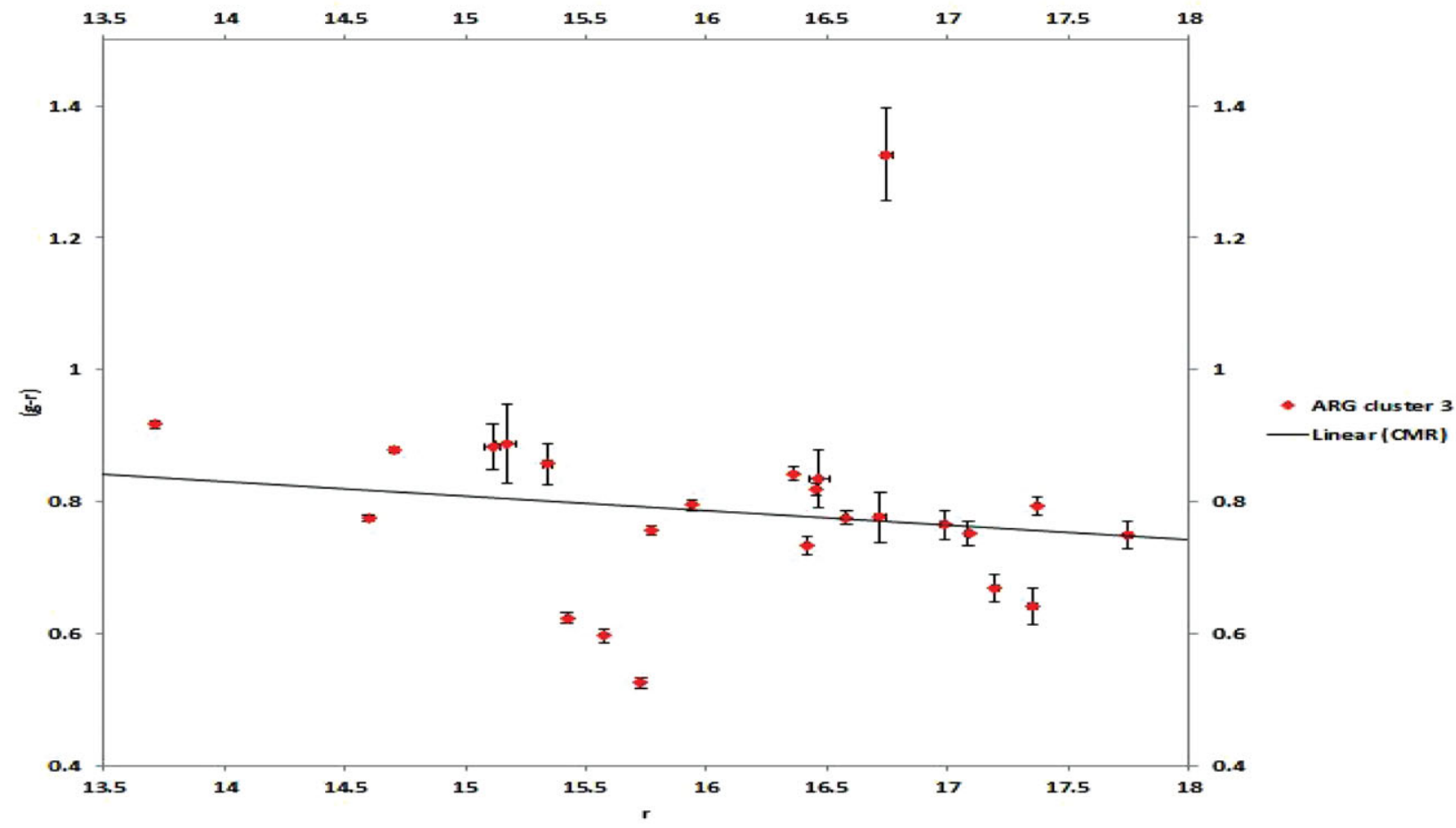

ARG 3

Figure 2. (continued). 
$\mathrm{RA}=200.90364, \mathrm{DEC}=4.67479, \mathrm{MJD}=52376$, Plate $=851$, Fiber $=23$

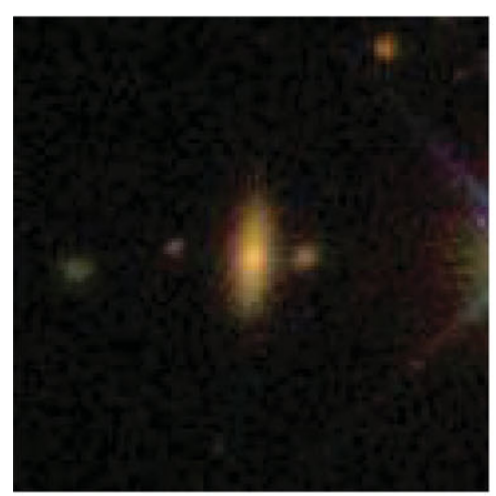

image 4

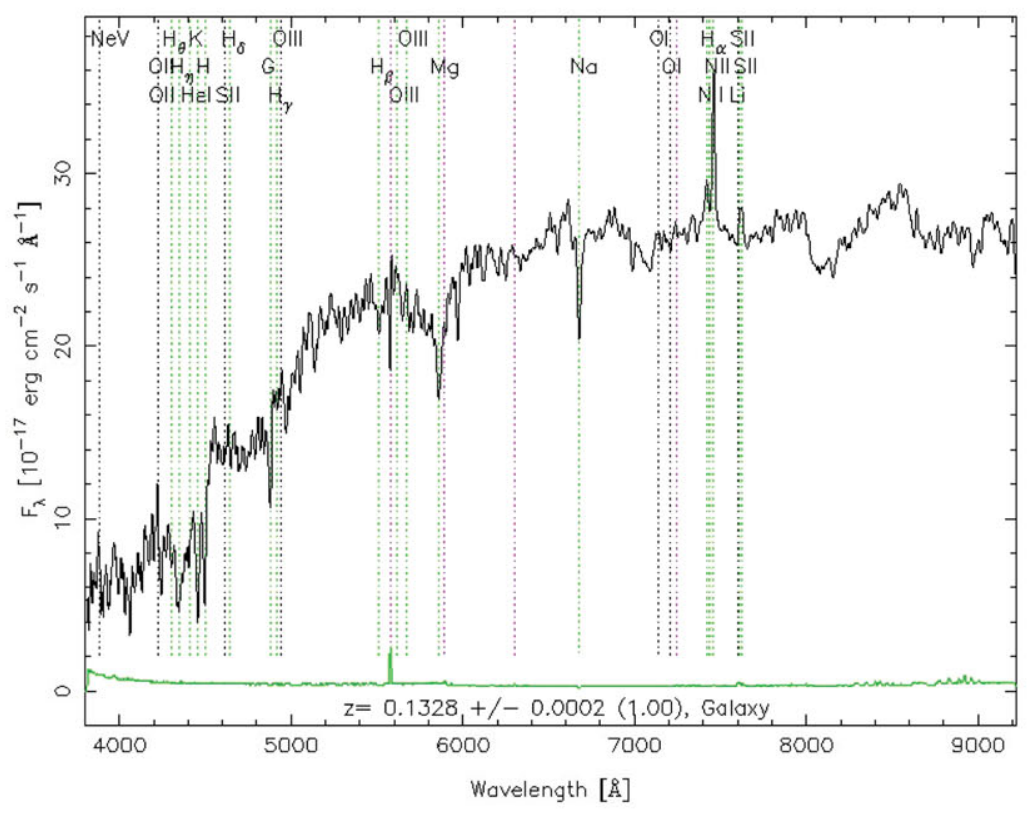

spectrum 4

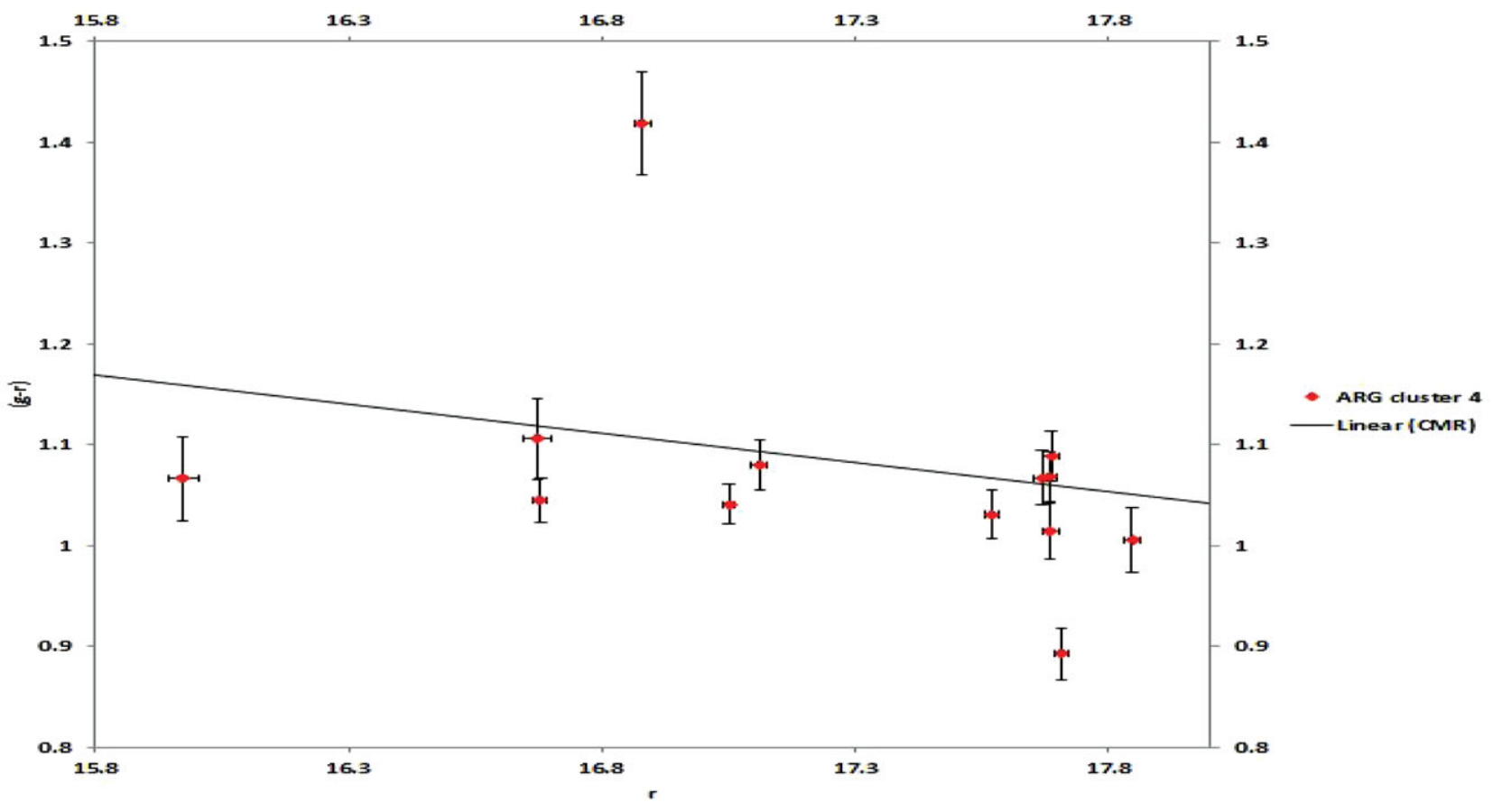

ARG 4

Figure 2. (continued). 


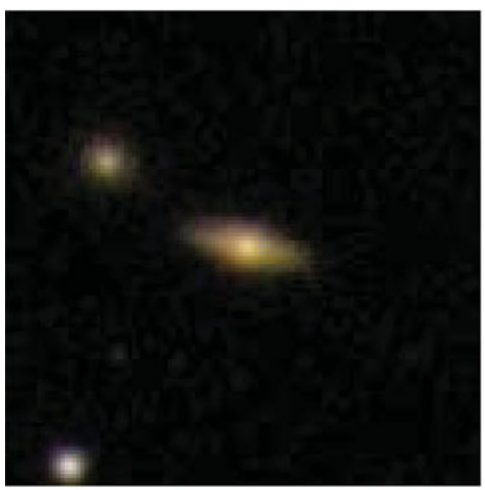

image 5
$\mathrm{RA}=258.79006, \mathrm{DEC}=64.33621, \mathrm{MJD}=51691$, Plate $=350$, Fiber $=165$

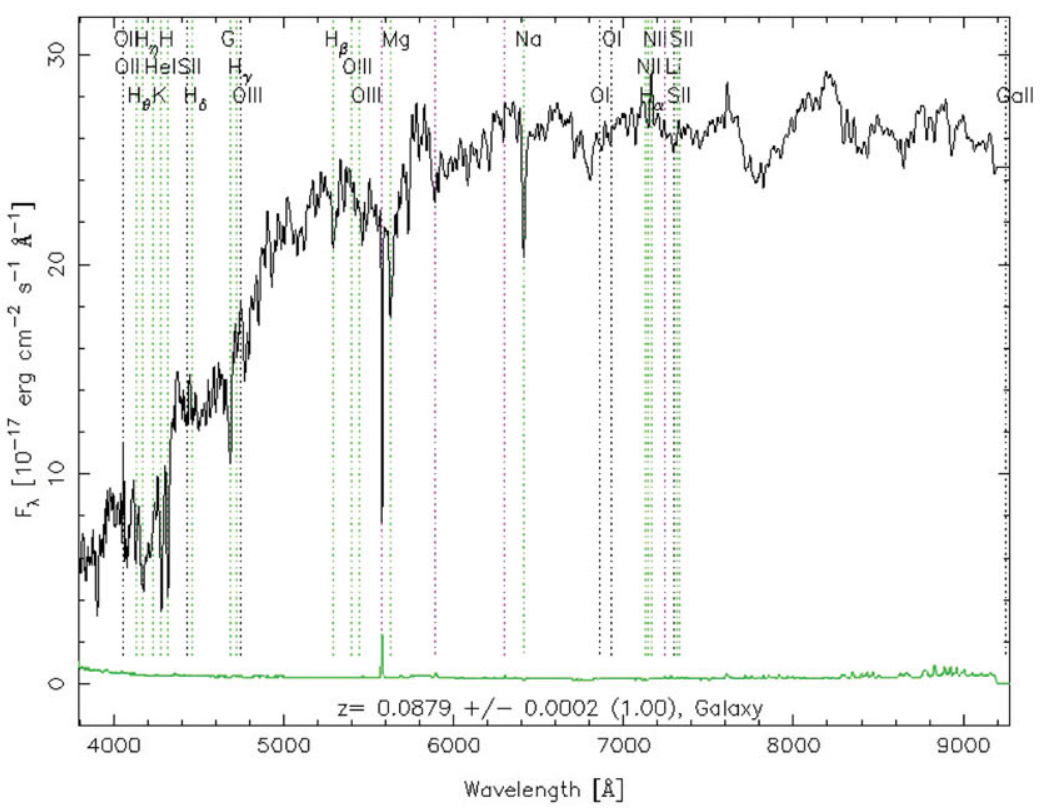

spectrum 5

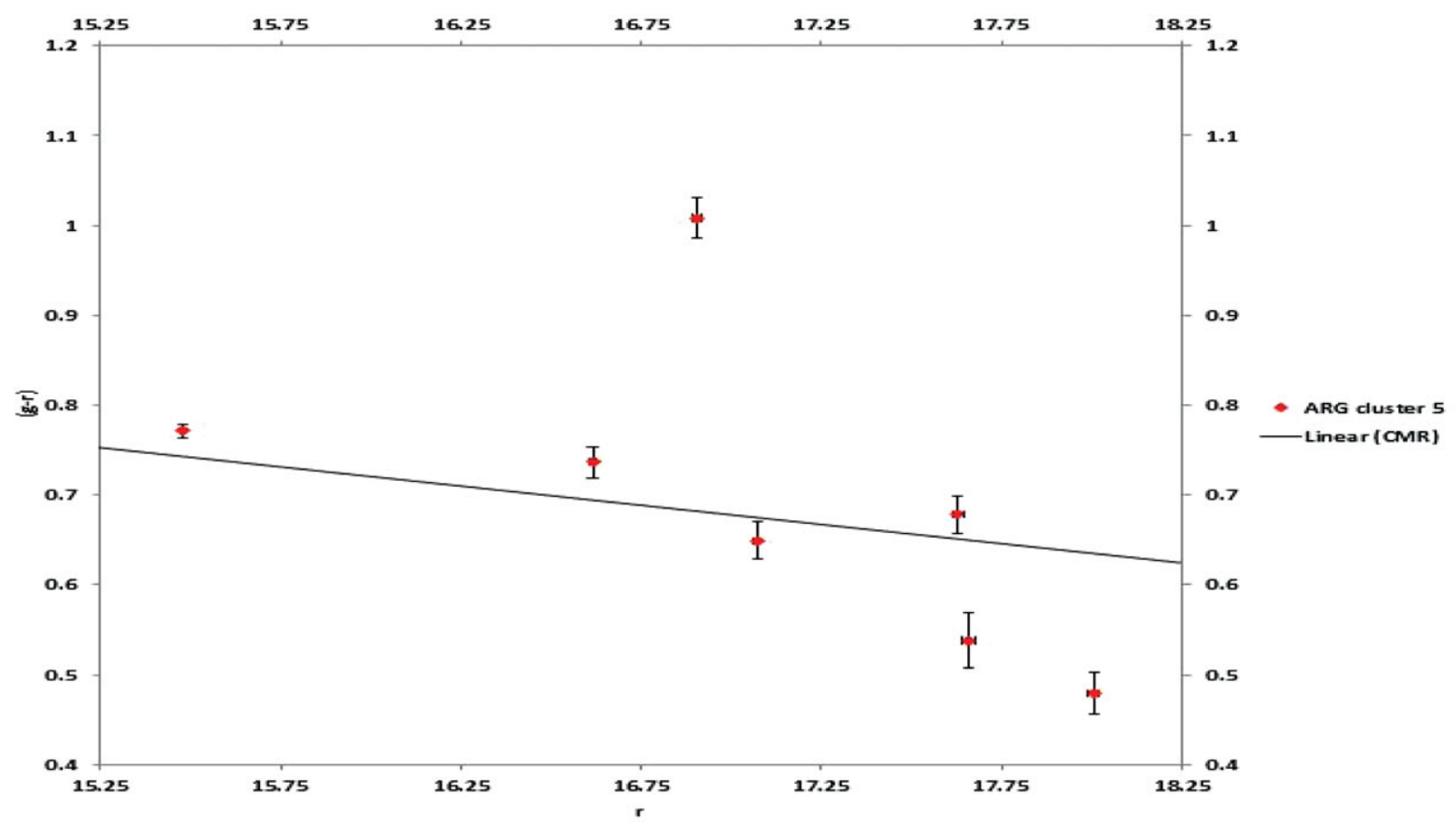

ARG 5

Figure 2. (continued). 


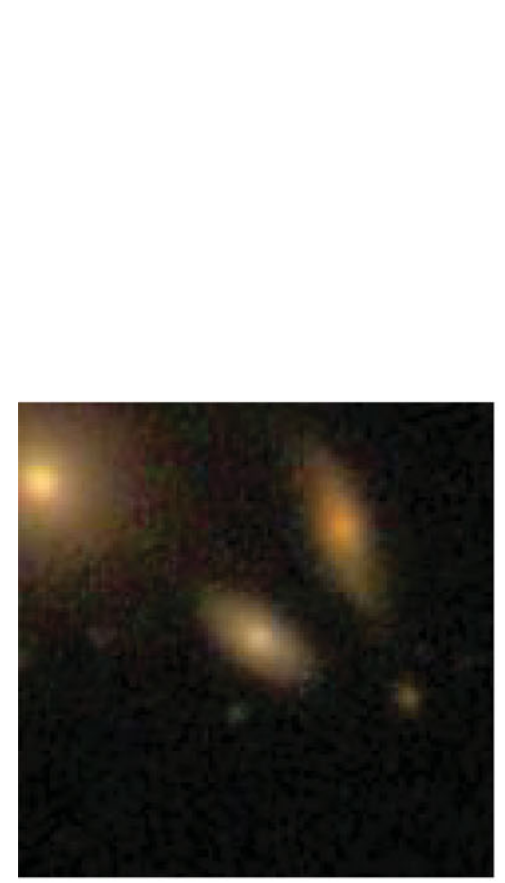

image 6

$R A=255.47875, D E C=59.57686, M J D=51703$, Plate $=353$, Fiber $=396$

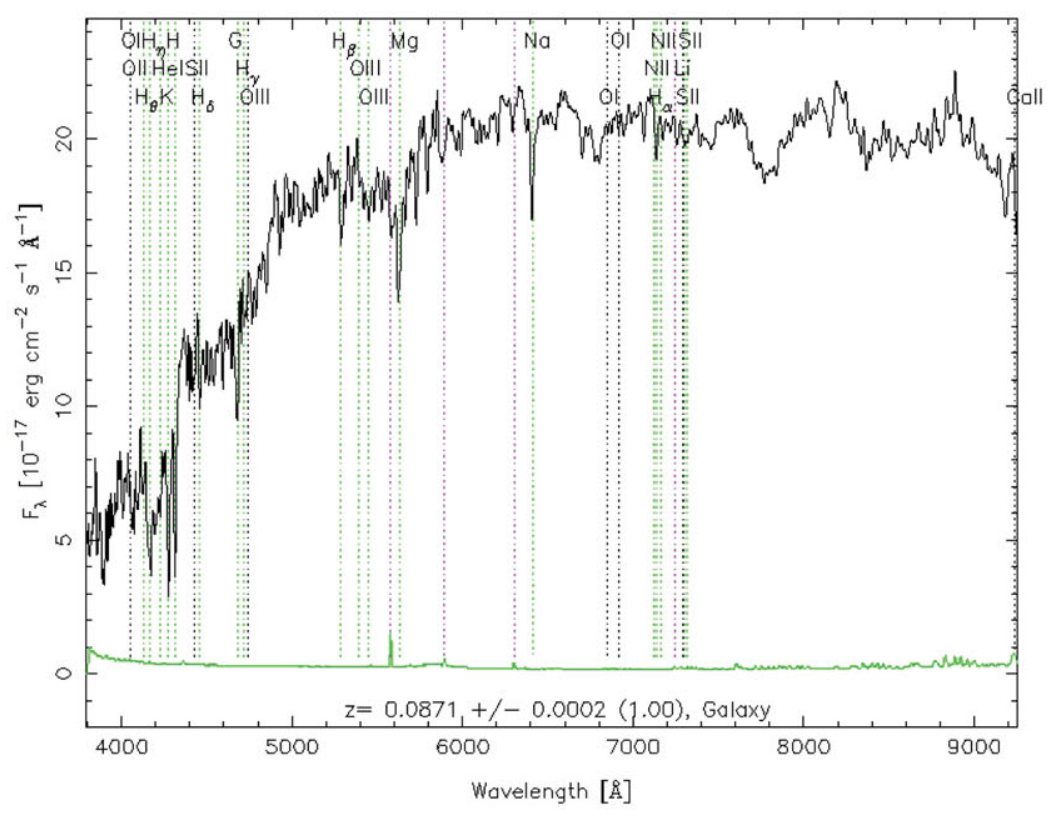

spectrum 6

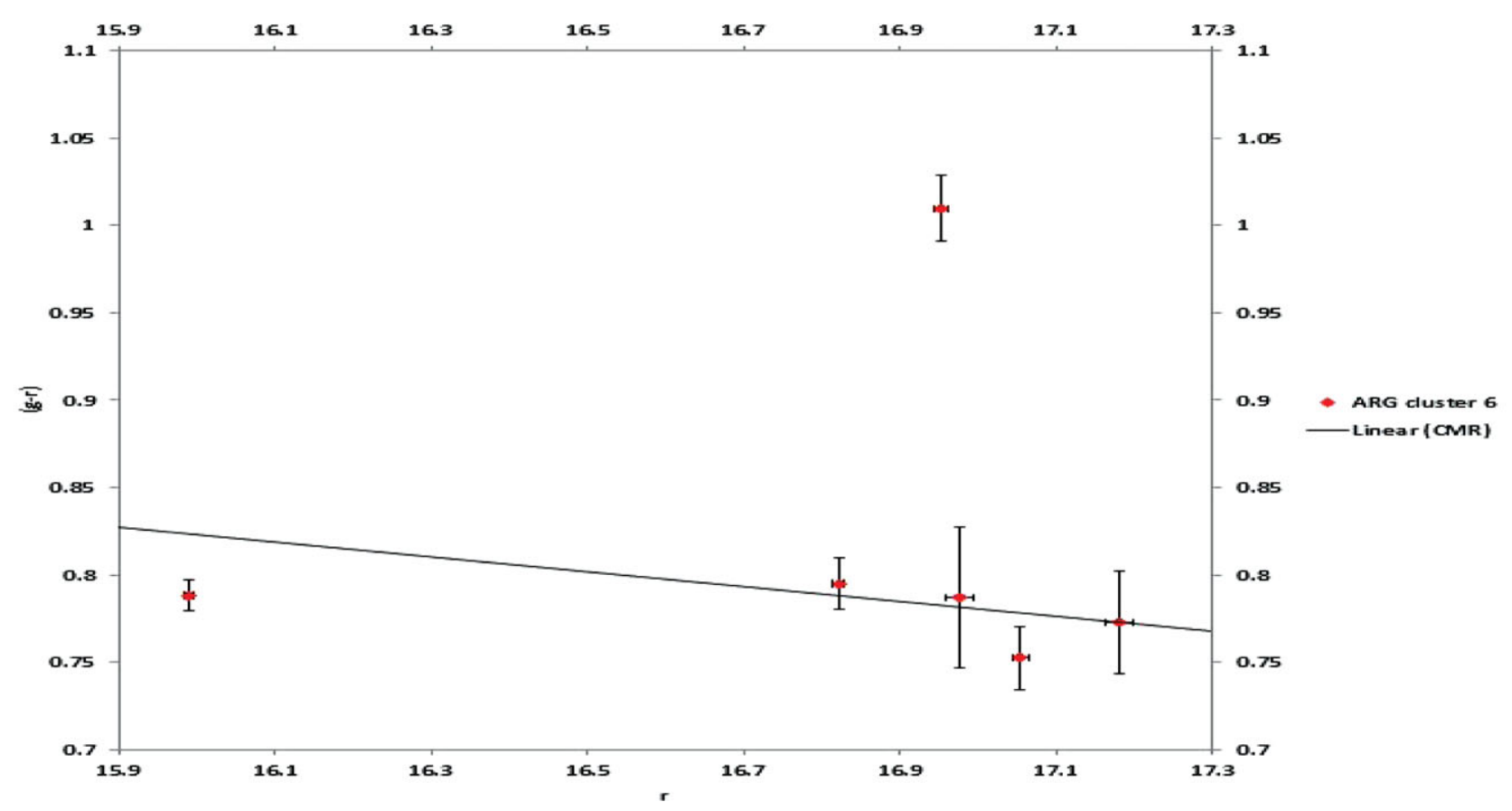

ARG 6

Figure 2. (continued). 
$\mathrm{RA}=134.15199, \mathrm{DEC}=53.52232, \mathrm{MJD}=51900$, Plate $=449$, Fiber $=182$

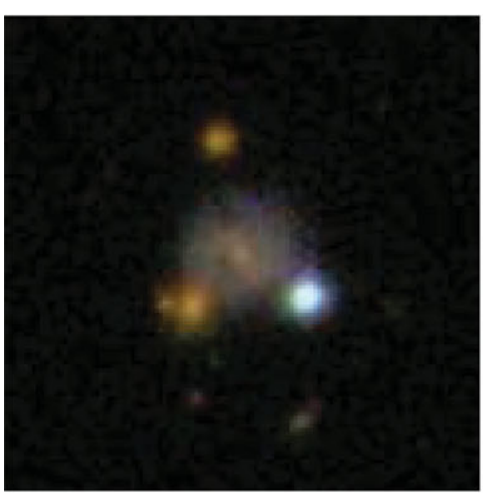

image 7

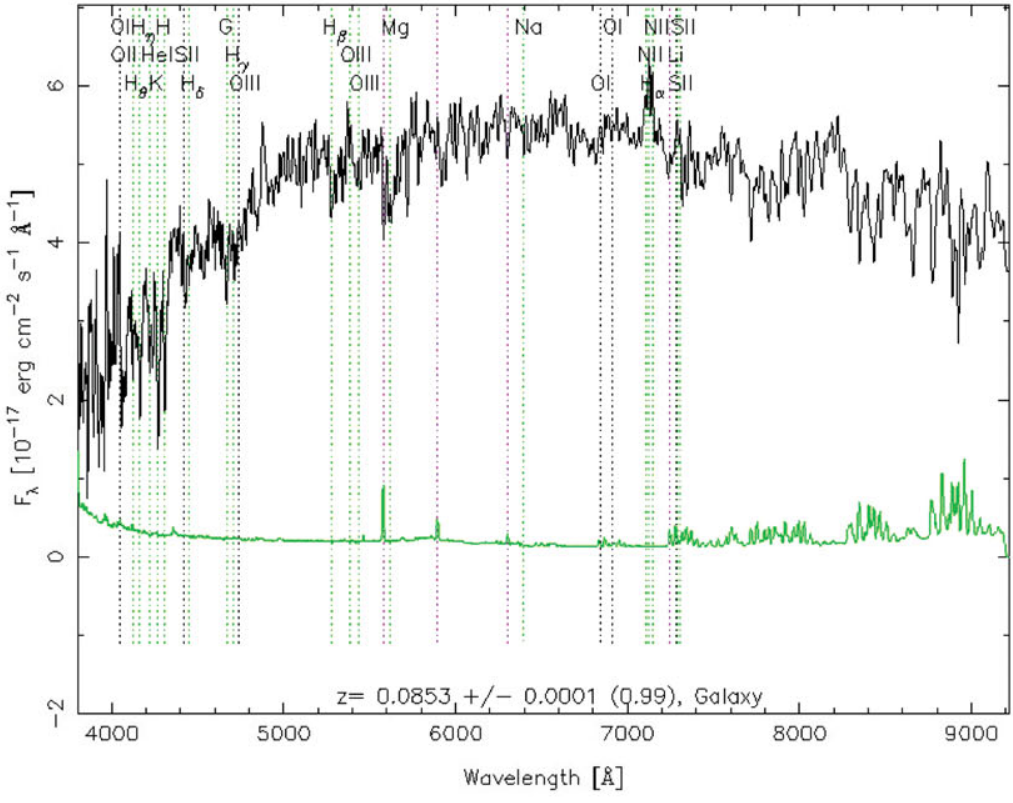

spectrum 7

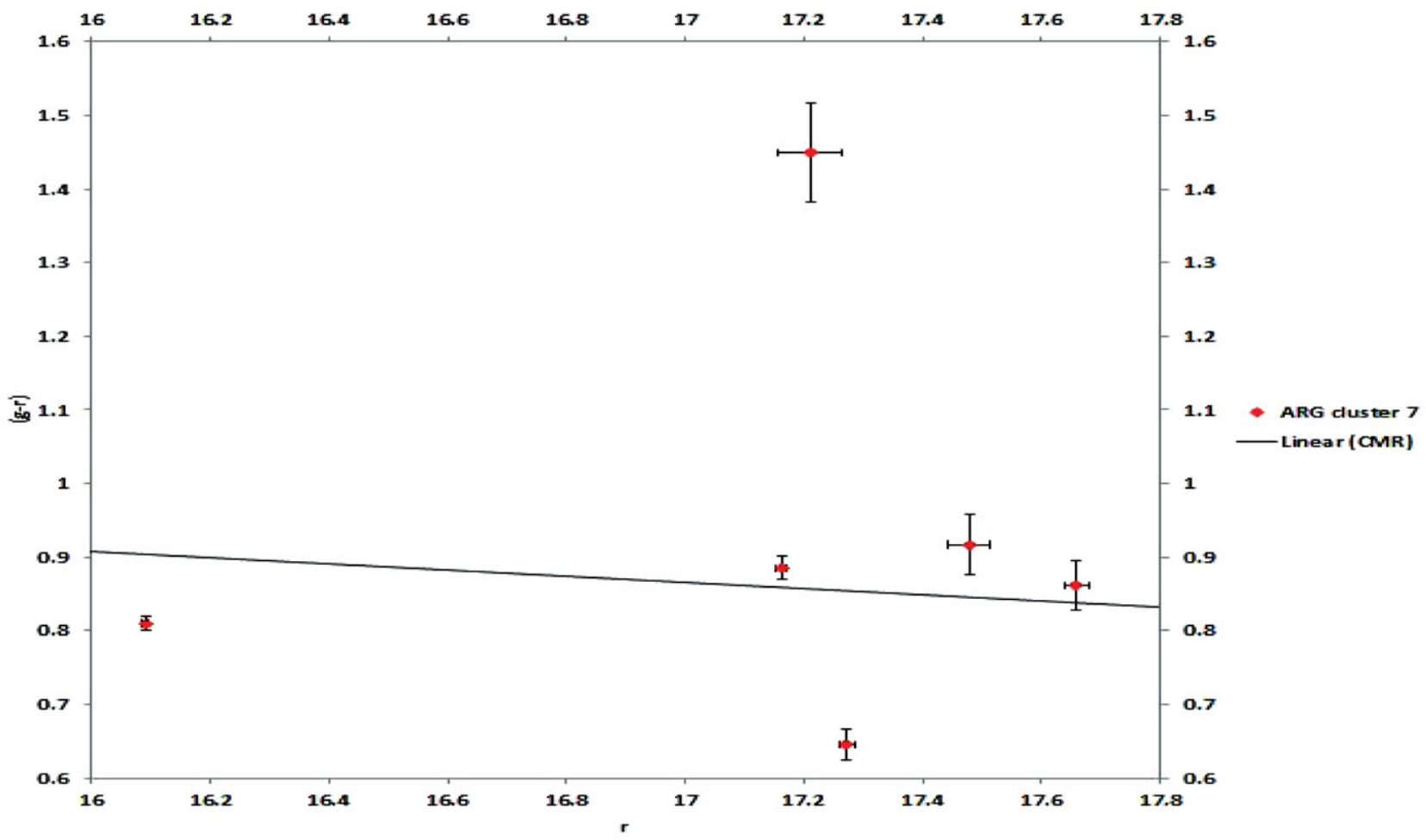

ARG 7

Figure 2. (continued). 
Table 1. ARG data.

\begin{tabular}{|c|c|c|c|c|c|c|c|}
\hline ARG ID & R.A & Dec & r mag & abs. r mag & redshift (z) & SDSS-C4 Parent cluster & Other identification \\
\hline 1 & 208.2798 & 5.1554 & 17.40 & -20.21 & 0.079742 & 1001 & Abell 1809 \\
\hline 2 & 118.6592 & 39.1774 & 17.39 & -20.61 & 0.095647 & 3168 & Abell 0607 \\
\hline 3 & 18.8185 & 0.1887 & 16.75 & -19.62 & 0.044938 & 2013 & Abell 0168 \\
\hline 4 & 200.9036 & 4.6748 & 16.89 & -21.84 & 0.132832 & 1091 & - \\
\hline 5 & 258.7901 & 64.3362 & 16.91 & -20.91 & 0.087914 & 3010 & - \\
\hline 6 & 255.4788 & 59.5769 & 16.95 & -20.85 & 0.087059 & 3160 & ZwCL $1700.2+5941$ \\
\hline 7 & 134.1520 & 53.5223 & 17.21 & -20.54 & 0.085262 & 3150 & - \\
\hline
\end{tabular}

$z=0.1$ (and only considering clusters below that redshift) yields a limit of $r=-20.33$ and comprises 4 ARGs, which is 0.0011 per cent of all galaxies below $z=0.1$ and brighter than this limit.

In summary, this work has:

(i) undertaken the first and largest systematic search for anomalously red cluster galaxies at low (SDSS) redshifts;

(ii) found seven galaxies out of 7485 that have colours significantly redder than the fitted CMR, accounting for photometric errors;

(iii) found that nearly a third of ARGs are caused by photometric contamination, but a subset of truly red cluster galaxies exist ( 0.0668 per cent of the cluster galaxy population). These can be roughly divided in to dusty starforming galaxies, and elliptical galaxies that probably possess a high dust content.

\section{ACKNOWLEDGEMENTS}

We thank Peter Jensen for pointing out the set of anomalous objects discussed in Jensen \& Pimbblet (2012) that inspired the present work. KAP thanks Christ Church College, Oxford, for their hospitality whilst some of this work was being undertaken. We also thank Benjamin Milill and Heath Jones for their assistance. We would like to express our gratitude to the anonymous referee for their feedback and patience.

Funding for the SDSS and SDSS-II has been provided by the Alfred P. Sloan Foundation, the Participating Institutions, the National Science Foundation, the U.S. Department of Energy, the National Aeronautics and Space Administration, the Japanese Monbukagakusho, the Max Planck Society, and the Higher Education Funding Council for England. The SDSS Web Site is http://www. sdss.org/.

The SDSS is managed by the Astrophysical Research Consortium for the Participating Institutions. The Participating Institutions are the American Museum of Natural History, Astrophysical Institute Potsdam, University of Basel, University of Cambridge, Case Western Reserve University, University of Chicago, Drexel University, Fermilab, the Institute for Advanced Study, the Japan Participation Group, Johns Hopkins University, the Joint Institute for Nuclear Astrophysics, the Kavli Institute for Particle Astrophysics and Cosmology, the Korean Scientist Group, the Chinese Academy of Sciences (LAMOST), Los Alamos National Laboratory, the Max-Planck-Institute for Astronomy (MPIA), the Max-
Planck-Institute for Astrophysics (MPA), New Mexico State University, Ohio State University, University of Pittsburgh, University of Portsmouth, Princeton University, the United States Naval Observatory, and the University of Washington.

\section{References}

Abazajian, K. N., et al. 2009, ApJS, 182, 543

Alpaslan, M., et al. 2012, MNRAS, 426, 2832

Beers, T. C., Flynn, K., \& Gebhardt, K., 1990, AJ, 100, 32

Bower, R. G., Lucey, J. R., \& Ellis, R. S., 1992, MNRAS, 254, 601

Brinchmann, J., Charlot, S., White, M., Tremonti, C., Kauffmann, G., Heckman, T., \& Brinkmann, J., 2004, MNRAS, 351, 1151

Cortese, L., 2012, A\&A, 543, A132

Cortese, L., \& Hughes, T. M. 2009, MNRAS, 400, 1225

Crossett, J. P., Pimbblet, K. A., Stott, J. P., \& Jones, D. H. 2013, MNRAS, submitted

Deng, X.-F., Chen, Y.-Q., \& Jiang, P., 2011, MNRAS, 417, 453

Goto, T., Yamauchi, C., Fujita, Y., Okamura, S., Sekiguchi, M., Smail, I., Bernardi, M., \& Gomez, P. L., 2003, MNRAS, 346, 601

Jensen, P. C., \& Pimbblet, K. A., 2012, MNRAS, 422, 2841

Ko, J., Hwang, H. S., Lee, J. C., \& Sohn, Y.-J., 2013, ApJ, 767, 90

Kodama, T., \& Arimoto, N., 1997, A\&A, 320, 41

Kodama, T., Bower, R. G., \& Bell, E. F., 1999, MNRAS, 306, 561

Lee, H., Bell, E. F., \& Somerville, R. S., 2008, in Low-Metallicity Star Formation: From the First Stars to Dwarf Galaxies, Proceedings of the International Astronomical Union, IAU Symposium, 255,100

Mahajan, S., \& Raychaudhury, S., 2009, MNRAS, 400, 687

Masters, K. L., et al. 2010a, MNRAS, 405, 783

Masters, K. L., et al. 2010b, MNRAS, 404, 792

Miller, C. J., et al. 2005, AJ, 130, 968

Poggianti, B. M., Smail, I., Dressler, A., Couch, W. J., Barger, A. J., Butcher, H., Ellis, R. S., \& Oemler, A. Jr, 1999, ApJ, 518, 576

Pimbblet, K. A., Smail, I., Edge, A. C., O’Hely, E., Couch, W. J., \& Zabludoff, A. I., 2006, MNRAS, 366, 645

Pimbblet, K. A., Smail, I., Kodama, T., Couch, W. J., Edge, A. C., Zabludoff, A. I., \& O’Hely, E., 2002, MNRAS, 331, 333

Robotham, A. S. G., et al. 2011, MNRAS, 416, 2640

Ruel, J., Bazin, G., Bayliss, M., Brodwin, M., Foley, R. J., Stalder, B., Aird, K. A., \& Armstrong, R., 2013, ApJ, 1131, 4953

Schlegel, D. J., Finkbeiner, D. P., Davis, M., 1998, ApJ, 500, 525

Terlevich, A. I., Kuntschner, H., Bower, R. G., Caldwell, N., Sharples, R. M., 1999, MNRAS, 310, 445

Visvanathan, N., \& Sandage, A., 1977, ApJ, 216, 214

Wolf, C., et al. 2009, MNRAS, 393, 1302

Worthey, G. 1994, ApJS, 95, 107 